\title{
Comportamiento de la eficiencia de los gobiernos locales de Junín mediante el análisis envolvente de datos
}

\author{
Efficiency behavior of local governments in Junin using data \\ envelopment analysis
}

\author{
Ybnias Elí Grijalva Yauri' \\ Universidad Continental \\ ygrijalva@continental.edu.pe
}

\section{RESUMEN}

La investigación comprende el análisis de los niveles de eficiencia de los gobiernos locales de la región Junín, período 2009 - 2014, mediante el método de análisis envolvente de datos (DEA) y el índice de Malmquist, que se aplican buscando el objetivo de identificar un conjunto de unidades de eficiencia que sirvan de referente a los gobiernos locales de la región y así impulsar el cumplimiento de la misión institucional y de las competencias de gobierno local y por ende promover el desarrollo integral de su jurisdicción. La hipótesis planteada fue que los gobiernos locales que reporten niveles de eficiencia alcanzarán el cumplimiento de su misión institucional y sus competencias así como el cumplimiento de sus funciones, logrando promover el desarrollo integral de su jurisdicción. Los resultados obtenidos, en función de tres competencias de los gobiernos locales (servicios públicos locales; organización del espacio físico y uso del suelo; servicios sociales locales), indican que los gobiernos locales de Junín no alcanzan niveles de eficiencia permanentes en el tiempo. En conclusión, no es posible identificar unidades de toma de decisiones (DMU) de eficiencia que sirvan como referencia a los gobiernos locales de Junín, limitando el cumplimiento de su misión institucional y cabal desempeño de competencias y funciones, en desmedro del desarrollo económico y social de su jurisdicción.

Palabras clave: Eficiencia, gobierno local, análisis envolvente de datos, índice Malmquist.

\begin{abstract}
The research includes the Efficiency Analysis of local governments in Junin Region (2009 - 2014 period), using the Data Envelopment Analysis - DEA method and Malmquist Index which is applied to "Identify a efficiency unit set that serve as a reference for local governments in the region, to promote compliance with the institutional mission and local government competences, and consequently, promote the integral development of its jurisdiction". The working hypothesis was: "Local governments that report efficiency levels will reach the fulfillment of its mission and its competences as well as their duties' performance, successfully promoting the integral development of its jurisdiction." The achieved results, applied to three local governments competences -local public services; physical space organization and land use; local social services- indicate that Junin local governments do not reach permanent efficiency levels over time. Finally, it is not possible to identify efficiency Decision Making Units (DMU) that serve as a reference for Junin local governments, thus, limiting the fulfillment of its institutional mission and proper functions and duties' performance, adding to the economic and social development detriment of its jurisdiction.
\end{abstract}

Keywords: Efficiency, local governments, data envelopment analysis, Malmquist index.

Historial del artículo:

Recibido: 4 de julio de 2015. Aprobado: 23 de octubre de 2015. Disponible en línea: 30 de diciembre de 2015

1 Economista, Ms. en Proyectos, docente de la Universidad Continental. 


\section{INTRODUCCIÓN}

La idea de eficiencia posee actualmente un gran arraigo e impulso en el sector público, por lo que se ha convertido en un esencial objetivo para los gobiernos locales. La necesidad de considerar el uso eficiente de los recursos es un objetivo explícitamente ya establecido para tales gobiernos.

En la literatura especializada, existe un creciente e importante número de estudios sobre la medida de la eficiencia, utilizando técnicas no paramétricas. Dos estudios podemos destacar como antecedentes: Chediak (1), La técnica DEA para medir y analizar la eficiencia de los municipios del departamento del Tolima (Colombia), en el 2006, cuyo objetivo fue establecer y analizar el ordenamiento de la eficiencia de estos municipios para evaluar la eficiencia en el desempeño local y establecer las mejoras potenciales. Según el modelo, el resultado se calcula empleando el método de DEA, el que permite conocer los municipios eficientes de referencia y las mejoras potenciales para cada municipio no eficiente, en qué proporción deben incrementar sus insumos y/o productos para alcanzar la eficiencia.

El otro estudio es del MINDES $(2,3)$, referido al análisis de eficiencia del Programa Nacional Wawa Wasi a nivel de sedes zonales, período 2007, 2008 y 2009, y cuyo objetivo fue estimar la eficiencia y productividad de las respectivas sedes mediante el método no paramétrico DEA y el índice Malmquist. Esto debido a que los métodos no paramétricos de análisis de eficiencia son consideradas como las alternativas más adecuadas para medir la eficiencia de las entidades del sector público, ya que en su aplicación se usan inputs y outputs, fácilmente calculables para este tipo de entidades.

El problema en la gestión de los gobiernos locales se caracteriza por la insatisfacción y el descontento de sus acciones, tanto políticos como ciudadanos, e incluso por los mismos servidores públicos, que cuestionan con frases como gestión improvisada, demasiado lenta, burocracia, demasiado alejado de las necesidades de las personas, con alto grado de corrupción, de mala calidad en sus servicios públicos, sin capacidad de gasto teniendo los recursos financieros necesarios y derroche de los recursos financieros.

El objetivo de la investigación fue identificar un conjunto de unidades de eficiencia que sirvan de referente a los gobiernos locales de la región y así impulsar el cumplimiento de la misión institucional y de las competencias de gobierno local y por ende promover el desarrollo integral de su jurisdicción. La hipótesis fue que los gobiernos locales que reporten niveles de eficiencia alcanzarán el cumplimiento de su misión institucional y sus competencias así como el cumplimiento de sus funciones, logrando promover el desarrollo integral de su jurisdicción.

El Perú ha mantenido una tasa de crecimiento económico (4), que va desde el $9 \%$ en el 2007 hasta el $5,8 \%$ en el 2013 , ritmo que se ve reflejado en los niveles de presupuesto destinados a los gobiernos locales de Junín. Los presupuestos de estos gobiernos han experimentado un crecimiento no antes visto $y$ muy difícil que se repita de 357 millones de nuevos soles en el 2007 a 1,098 millones en el 2013. Particularmente, los presupuestos para inversiones presentaron un singular crecimiento, de 202 millones de nuevos soles en el 2007 a 668 millones en el 2013.

El rápido crecimiento presupuestario de los gobiernos locales de Junín lleva a plantearse una serie de interrogantes que tienen que ver con la eficiencia con que se administran dichos recursos por parte de las autoridades. Los estudios de eficiencia están relacionados con funciones de producción, y para su estudio en primer instante se debe diferenciar entre los métodos paramétricos y los métodos no paramétricos. La principal diferencia entre ellos está relacionada por la capacidad de formular y establecer la forma funcional de las funciones de producción y las situaciones de multiproducto.

Los gobiernos locales producen bienes y servicios en mercados que funcionan parcialmente o no funcionan; carecen de un sistema automático de incentivos por precios o por competencia para mejorar la asignación de recursos; los costos de oportunidad son altos por la escasez de los recursos. En este contexto, se hace viable medir los niveles de eficiencia de los gobiernos locales de Junín, como una medida de desempeño de la gestión pública recurriendo a los métodos no paramétricos.

En los métodos no paramétricos, el más utilizado es el análisis envolvente de datos (DEA), que en conjunto con el índice de Malmquist permite medir los niveles de eficiencia a través de tiempo de las unidades de toma de decisión (DMU) (gobiernos locales). Dicho proceso consiste en encontrar un conjunto de unidades eficientes y a partir de su combinación lineal, generar una envolvente o frontera eficiente.

El objetivo del DEA es optimizar la eficiencia relativa de cada DMU, para establecer una frontera de eficiencia, usando el criterio de eficiencia de Pareto. La frontera eficiente está conformada por aquellas DMU eficientes. El índice de Malmquist consiste en el cálculo de índices a partir de funciones de distancia. Usando dichas funciones de distancia, establece en qué medida una DMU es eficiente o ineficiente. 


\section{MATERIAL Y MÉTODOS}

La investigación por su finalidad es explicativa. Utiliza un diseño no experimental de panel de datos, con los gobiernos locales seleccionados en el período 2009 - 2014, y emplea el índice de Malmquist mediante el análisis envolvente de datos (DEA).

El estudio considera las siguientes competencias de los gobiernos locales, para las que se estimarán una función de producción correspondiente: (5) servicios públicos locales (recolección de residuos sólidos); organización del espacio físico y uso del suelo (pistas y veredas, licencias de construcción); y servicios sociales locales (programas sociales y seguridad ciudadana).

Según su organización política, Junín tiene 123 distritos, agrupados en 9 provincias. La muestra considera dos indicadores relevantes: población distrital anual, y asignación presupuestal anual. Establecidos los promedios para 2009 - 2014, con un ordenamiento descendente se obtiene la muestra de 26 gobiernos locales.

La técnica de recolección de datos se da mediante el análisis de contenidos. La información está centralizada en el INEI a través del Registro Nacional de Municipalidades (RENAMU), encuestas obligatorias anuales, y en el MEF, en su Portal de Transparencia Económica.

\section{Aspectos legales de los gobiernos locales}

La Ley Orgánica de Municipalidades (5), Ley Nº 27972, señala en su artículo IV, Título Preliminar, que los gobiernos locales tienen la finalidad de "representar al vecindario, promover la adecuada prestación de los servicios públicos locales y el desarrollo integral, sostenible y armónico de su circunscripción".

Esta ley identifica siete áreas en las que los gobiernos locales deben intervenir y actuar: 1) organización del espacio físico - uso del suelo; 2) servicios públicos locales; 3) protección y conservación del ambiente; 4) desarrollo y economía local; 5) participación vecinal; 6) servicios sociales locales; y 7) prevención, rehabilitación y lucha contra el consumo de drogas.

\section{Concepto general de eficiencia}

La propuesta es "recurrir al uso de 'cónicas o poligonales convexas' para construir isocuantas o fronteras, en forma no paramétrica, y solo partiendo de la información disponible acerca del comportamiento de numerosas DMU comparables, muchas de las cuales serán más eficientes que otras" (6).

\section{Análisis envolvente de datos (DEA)}

La medición de la eficiencia tiene sus inicios en el trabajo de Farrell, esfuerzo que es complementado con los trabajos de Charmes, Cooper y Rhodes (CCR) que partían de rendimientos a escala constante. Banker, Cooper y Rhodes (BCC) desarrollan el modelo original incluyendo los rendimientos a escala variable. Esta expansión del modelo permitió que la unidad evaluada fuese comparada con unidades de similar tamaño $(7,8)$.

\section{Orientación del modelo DEA}

La "orientación" está relacionada con la forma que se busca mejorar los índices de eficiencia: $(1,9)$

- Orientados a inputs: Buscan, dado el nivel de outputs, la máxima reducción en el vector de inputs. Una unidad no es eficiente si es posible disminuir cualquier input sin alterar sus outputs.

- Orientados a outputs: Buscan, dado el nivel de inputs, el máximo incremento de los outputs permaneciendo dentro de la frontera de posibilidades de producción. Una unidad no puede ser caracterizada como eficiente si es posible incrementar cualquier output sin incrementar (disminuir) ningún otro output.

Análisis envolvente de datos (DEA) - modelo BCC - O: orientado al output con frontera eficiente a retornos de escala variable (1)

Forma dual para cada DMU:

Maximizar $\mathrm{Z}=\varphi+\varepsilon\left[\sum_{\mathrm{r}=1}^{\mathrm{t}} \mathrm{S}_{\mathrm{r}}^{+}+\sum_{\mathrm{i}=1}^{\mathrm{m}} \mathrm{S}_{\mathrm{r}}^{-}\right]$

sujeto a:

$\mathrm{Y}_{\mathrm{rj}} \varphi-\sum_{\mathrm{i}=1}^{\mathrm{m}} \mathrm{Y}_{\mathrm{ij}} \lambda_{\mathrm{j}}+\mathrm{S}_{\mathrm{r}}^{+} \leq 0, \mathrm{r}=1, \ldots, \mathrm{t}$

$\sum_{i=1}^{m} X_{i j} \lambda_{j}+S_{i}^{-} \leq X_{i j}, \quad i=1, \ldots, m$

$\sum_{i=1}^{m} V_{i} X_{i j}=1$

$\varphi$ : irrestricta o libre

$\lambda_{\mathrm{j}} \geq 0 ; \mathrm{j}=1, \ldots, \mathrm{n} ; \mathrm{S}_{\mathrm{r}}^{+} \geq 0 ; \mathrm{r}=1, \ldots, \mathrm{t} ; \mathrm{S}_{\mathrm{i}}^{-} \geq 0 ; \mathrm{i}=1, \ldots, \mathrm{m}$

Donde:

Número de inputs: $\mathrm{i}=1, \ldots, \mathrm{m}_{i} \sum_{(\mathrm{i}=1)}^{\mathrm{m}} \mathrm{v}_{\text {in }} \mathrm{X}_{\mathrm{in}}$; número

de outputs: $r=1, \ldots, t: \sum_{(r=1)}^{t} u_{r n} Y_{r n}$; número de DMU:

$\mathrm{j}=1, \ldots, \mathrm{n} ; \mathrm{DMU} \mathrm{n}$ 
$\varphi^{*}$ : Proporción de inputs actuales que deben utilizarse para alcanzar la eficiencia.

$\lambda_{j}^{*}$ : Vector columna cuyas componentes son multiplicadores adimensionales, miden la proximidad de la proyección resultante de cada DMU con las unidades eficientes de las que es combinación lineal.

$\mathrm{S}_{i}^{-*}$ : Vector columna de las variables de holgura correspondientes a las desigualdades de los inputs, sus unidades estarán expresadas según las variables inputs.

$\mathrm{S}_{\mathrm{r}}^{+*}$ : Vector columna de las unidades de holgura correspondientes a las desigualdades de los outputs, sus unidades están expresadas según sus variables de outputs.

\section{Evolución de la productividad con el índice de Malmquist}

El índice de Malmquist (10) consiste en el cálculo de índices a partir de funciones de distancia. Desde dichas funciones se podrá establecer en qué medida un DMU es eficiente, y en caso de no serlo, cómo es de ineficiente, en relación con una eficiencia óptima del mercado para esa DMU. El referido índice presenta ventajas frente a otros métodos tradicionales de medir la productividad global: primero, no se necesitan supuestos sobre el comportamiento de la unidad que se analiza, tales como la maximización de beneficios o la minimización de costos; segundo, el índice está basado en funciones de distancia, por lo que no se requieren precios de inputs $u$ outputs en su construcción.

Utilizando la programación lineal DEA (basada en inputs $u$ outputs) para medir el cambio de la productividad, a través del índice de Malmquist, se especifica un índice basado en el output del cambio de la productividad de Malmquist como:

$m_{o}\left(y_{t+1}, x_{t+1}, y_{t}, x_{t}\right)=\left(\frac{d_{o}^{t}\left(x_{t+1}, y_{t+1}\right)}{d_{o}^{t}\left(x_{t}, y_{t}\right)} \frac{\left(d_{o}^{(t+1)}\left(x_{t+1}, y_{t+1}\right)\right.}{d_{o}^{t+1}\left(x_{t}, y_{t}\right)}\right)^{1 / 2}$

El problema de PL utilizado para el cálculo de la distancia $\mathrm{d}_{\mathrm{o}}^{\mathrm{t}}\left(\mathrm{x}_{\mathrm{t}}, \mathrm{y}_{\mathrm{t}}\right)$ exceptúa la restricción de convexidad y se incluye el (subíndice de) tiempo. Es decir:

$\left[\mathrm{d}_{\mathrm{o}}^{\mathrm{t}}\left(\mathrm{x}_{\mathrm{t}}, \mathrm{y}_{\mathrm{t}}\right)\right]^{-1}=\max _{\phi, \lambda} \phi$

Sujeto a:

$$
\begin{gathered}
-\phi \mathrm{y}_{\mathrm{i}, \mathrm{t}}+\mathrm{Y}_{\mathrm{t}} \lambda \geq 0 \\
\phi \mathrm{x}_{\mathrm{i}, \mathrm{t}}-\mathrm{X}_{\mathrm{t}} \lambda \geq 0 \\
\lambda \geq 0
\end{gathered}
$$

\section{RESULTADOS}

\section{Aplicación de los índices de Malmquist mediante el DEA a los gobiernos locales del departamento de Junín}

La eficiencia mide la relación entre productos obtenidos e insumos utilizados en un proceso productivo. Cualquier actividad productiva puede entenderse como un proceso de optimización, en el cual se busca alcanzar la máxima cantidad de productos sin desperdiciar, o aprovechar al máximo los recursos o insumos disponibles (11).

\section{Función de producción de los servicios públicos locales}

Los gobiernos locales deben cumplir con regular y controlar el proceso de recolección, disposición final de los desechos sólidos, líquidos y vertientes industriales

\begin{tabular}{|c|c|}
\hline Outputs & Inputs \\
\hline $\begin{array}{l}\text { Output 1: Residuos } \\
\text { sólidos } \\
\text { (toneladas } \\
\text { recolectadas por año). }\end{array}$ & $\begin{array}{l}\text { Inputs 1: Vehículos (destinados } \\
\text { a la recolección de residuos } \\
\text { sólidos). } \\
\text { Inputs 2: Costos que genera la } \\
\text { recolección de residuos sólidos } \\
\text { (nuevos soles). } \\
\text { Inputs 3: Obreros (número de } \\
\text { trabajadores destinados a la } \\
\text { recolección de residuos sólidos). }\end{array}$ \\
\hline
\end{tabular}

Tabla $\mathrm{N}^{\circ}$ 1: Variables para medir los niveles de eficiencia de los servicios públicos.

en su ámbito $(12,13,14)$. Asimismo, proveer del servicio de limpieza pública determinando las áreas de acumulación de desechos, rellenos sanitarios y el aprovechamiento industrial de desperdicios. La tabla 1 muestra las variables seleccionadas como inputs $u$ outputs, y que son consideradas pertinentes para la estimación de los niveles de eficiencia.

\section{Estimación del modelo}

Con las técnicas del DEA e índice de Malmquits se ha realizado estimaciones para los 26 gobiernos locales de la muestra, procedimiento a partir del cual se obtiene la solución mostrada en la tabla 2.

A partir de la tabla 2 se puede identificar los siguientes comportamientos:

- La evolución del índice de Malmquist presenta una alta dispersión, no existe gobierno local que registre durante el período más de un índice que indique continuidad en eficiencia del tratamiento de los 
residuos sólidos. Esto es una señal de que no existe una adecuada planificación de mediano y largo plazo en el manejo de los residuos sólidos.

- El gobierno local de Huancayo la función de recolección y disposición de residuos sólidos lo tiene concesionado a una empresa privada, pese a ello, no alcanza los niveles de eficiencia que se esperaría.

- Los gobiernos locales de El Tambo, Satipo, Chupaca, que tienen las mismas autoridades, deberían desarrollar procesos eficientes de tratamiento de
Durante el período analizado la gestión de residuos sólidos, se puede observar que:

- El gobierno local distrital de Sapallanga aparece con niveles de eficiencia en cuatro de un total de cinco períodos de comparación.

- Los gobiernos locales de Perené, Pangoa, Satipo, Chanchamayo, La Oroya, Río Tambo, Río Negro, Junín, Pilcomayo, aparecen en tres de un total de cinco oportunidades con niveles de eficiencia.

- Los gobiernos locales de Tarma, Pichanaqui,

Tabla N²: Indice de Malmquits - residuos sólidos (2009-2014).

\begin{tabular}{lrrrrr}
\hline Malmquist & $2009=>2010$ & $2010=>2011$ & $2011=>2012$ & $2012=>2013$ & $2013=>2014$ \\
\hline El Tambo & 0,93034054 & 3,11561951 & 0,86783771 & 0,59042315 & 0,68016554 \\
Huancayo & 0,96148650 & 0,74940683 & 0,99775533 & 0,84979402 & 0,44168042 \\
Chilca & 0,76233924 & 0,99518069 & 0,20447265 & 7,12130928 & 0,14444005 \\
Tarma & 2,18226756 & 1,33051738 & 0,43964232 & 0,63375014 & 0,39129325 \\
Perené & 1,00892875 & 10,72533200 & 0,02987746 & 51,44166030 & 0,26920349 \\
Pichanaqui & 0,52498742 & 0,80712516 & 1,24634687 & 0,71484419 & 1,01732725 \\
Pangoa & 1,77809001 & 1,62413130 & 1,15426655 & 0,95658606 & 0,93685569 \\
Satipo & 0,98098283 & 0,79538109 & 1,04646126 & 0,41871072 & 1,55485177 \\
Chanchamayo & 4,09681465 & 1,20110376 & 1,02298038 & 1,04402512 & 0,52927519 \\
San Ramón & 0,82832520 & 0,67682302 & 0,50204443 & 2,83831121 & 0,95735228 \\
Chupaca & 0,08028538 & 1,01838756 & 0,41140611 & 1,11305530 & 0,84446404 \\
La Oroya & 3,99343838 & 1,01238930 & 0,81746362 & 1,39099642 & 0,41401782 \\
Jauja & 0,85506391 & 1,05622377 & 0,82166620 & 2,26599426 & 0,73887123 \\
Río Tambo & 0,84744511 & 1,18001743 & 0,01219593 & 37,88940070 & 2,73908290 \\
Mazamari & 0,76908670 & 0,78223817 & 0,41050006 & 0,36505103 & 1,16866853 \\
Río Negro & 0,01000000 & 2,68623694 & 8,84603887 & 3,15580322 & 0,55169805 \\
Concepción & 0,83839440 & 2,54006397 & 1,00571067 & 1,18221679 & 0,64026847 \\
Junín & 1,75525284 & 1,65612013 & 0,01923939 & 21,88416990 & 0,61463423 \\
Sapallanga & 2,19052357 & 1,05020785 & 3,82136101 & 0,75897814 & 2,36533074 \\
Pilcomayo & 0,72243563 & 1,41533057 & 2,44264022 & 0,85108297 & 6,48535544 \\
Huancán & 0,82118490 & 0,42143002 & 4,61156772 & 0,24085349 & 5,42924067 \\
San Agustín & 0,52090561 & 0,52113630 & 7,92659770 & 0,84061575 & 0,27966639 \\
Yauli & 0,23571234 & 7,27142123 & 0,35220890 & 2,41704809 & 0,57489678 \\
Morococha & 0,01000000 & 3,28471867 & 2,05334421 & 0,34544445 & 0,93998896 \\
Huay-Huay & 0,01000000 & 0,22791789 & 1,87627608 & 0,26887150 & 11,06403960 \\
Santa Rosa de Sacco & 0,94690659 & 0,05609083 & 9,73047359 & 0,45391160 & 1,57164832 \\
\hline
\end{tabular}

los residuos sólidos, sin embargo, ninguno muestra índices de eficiencia.

- Los gobiernos locales capitales de provincias, manejan mayores niveles de presupuesto, tienen mayor número de personal y disponen de mayor infraestructura; pero tales ventajas no se evidencian en una gestión eficiente.

- El gobierno local de Concepción es el único que cuenta con un área destinado a la disposición final de los residuos sólidos.
Chupaca, Jauja, Concepción, Huancán, Yauli, Morococha, Huay-Huay, Santa Rosa de Sacco, aparecen en dos de un total de cinco oportunidades con niveles de eficiencia.

- Los gobiernos locales de San Ramón, Mazamari y San Agustín, aparecen en uno de un total de cinco oportunidades con niveles de eficiencia.

- Los gobiernos locales de El Tambo, Huancayo, Chilca, no aparecen con niveles de eficiencia. 
Tabla $N^{\circ}$ 3: Variables para medir los niveles de eficiencia de los servicios públicos.

\begin{tabular}{|c|c|}
\hline Outputs & Inputs \\
\hline $\begin{array}{l}\text { Output 1: Licencias de construcción } \\
\text { (número de licencias). }\end{array}$ & $\begin{array}{l}\text { Inputs 1: Maquinaria pesada (número de } \\
\text { unidades). }\end{array}$ \\
\hline $\begin{array}{l}\text { Output 2: Pistas y veredas } \\
\text { (construidas y reparadas en metros }\end{array}$ & $\begin{array}{l}\text { Inputs 2: Equipos de oficina (número de } \\
\text { unidades). }\end{array}$ \\
\hline cuadrados) & $\begin{array}{l}\text { Inputs 3: Personal (número de profesionales, } \\
\text { técnicos, auxiliares). } \\
\text { Inputs 4: Gasto en infraestructura (nuevos } \\
\text { soles). }\end{array}$ \\
\hline
\end{tabular}

Tabla N 4: Índice de Malmquist - licencia de construcción, construcción de pistas y veredas (2009-2014).

\begin{tabular}{|c|c|c|c|c|c|}
\hline Malmquist & $2009=>2010$ & $2010=>2011$ & $2011=>2012$ & $2012=>2013$ & $2013=>2014$ \\
\hline El Tambo & 0,91472068 & 1,47675193 & 0,80586514 & 1,85938620 & 0,87511556 \\
\hline Huancayo & 0,78502043 & 1,27780770 & 0,85798500 & 1,06795527 & 1,03232739 \\
\hline Chilca & 1,27316118 & 1,54289084 & 1,52642204 & 0,87599684 & 0,23695897 \\
\hline Tarma & 0,08264456 & 2,37232565 & 0,90031379 & 1,73185157 & 2,31314451 \\
\hline Perené & 0,65318904 & 11,70274100 & 0,46925153 & 0,53581338 & 0,23815196 \\
\hline Pichanaqui & 0,32047478 & 2,00889837 & 0,47130938 & 1,66293749 & 1,58837014 \\
\hline Pangoa & 0,24657185 & 18,65801210 & 1,40248336 & 2,99936424 & 1,57316019 \\
\hline Satipo & 0,45718472 & 2,41962561 & 1,32847509 & 0,57685698 & 0,41960058 \\
\hline Chanchamayo & 1,56786013 & 1,61990398 & 2,87052211 & 0,59201854 & 1,04291960 \\
\hline San Ramón & 0,51072547 & 1,73626772 & 0,69581260 & 1,53206960 & 1,19996176 \\
\hline Chupaca & 0,32480039 & 3,85205307 & 0,95179125 & 1,41353906 & 0,23915221 \\
\hline La Oroya & 9,17439653 & 0,81792790 & 0,30136728 & 0,51850596 & 0,95373877 \\
\hline Jauja & 2,49306551 & 0,97707639 & 0,14598644 & 2,71123792 & 49,37221900 \\
\hline Río Tambo & 0,45900825 & 2,62795470 & 0,17246869 & 0,48970217 & 100 \\
\hline Mazamari & 0,24801459 & 4,60824953 & 1,53182444 & 0,09587315 & 0,02114844 \\
\hline Río Negro & 3,16521196 & 1,22234263 & 1,54662744 & 0,02909412 & 100 \\
\hline Concepción & 2,82819007 & 0,52177919 & 0,37649993 & 5,81620016 & 0,38977166 \\
\hline Junín & 0,01089864 & 93,87517880 & 0,94150135 & 0,83171983 & 3,76935477 \\
\hline Sapallanga & 0,17213305 & 33,98564500 & 1,53037338 & 3,89284891 & 7,91698679 \\
\hline Pilcomayo & 3,28978449 & 1,10988497 & 1,25465245 & 0,01376128 & 65,81083340 \\
\hline Huancán & 1,16523759 & 3,61098811 & 2,96494069 & 0,32119453 & 0,60678540 \\
\hline San Agustín & 0,42922472 & 2,37828896 & 2,53279625 & 1,24169647 & 0,35279088 \\
\hline Yauli & 0,93149967 & 12,72728720 & 0,17400483 & 0,53859882 & 0,27726505 \\
\hline Morococha & 2,61930679 & 0,02471007 & 100 & 0,02256298 & 5,10979191 \\
\hline Huay-Huay & 0,29378963 & 1,72192080 & 0,37811162 & 1,88379764 & 0,64991561 \\
\hline Santa Rosa de Sacco & 1,37422291 & 1,85530076 & 0,70021387 & 1,09769427 & 2,18162068 \\
\hline
\end{tabular}

\section{Función de producción de organización del espacio físico y uso del suelo}

En esta competencia deben cumplir con otorgar las licencias de construcción, bajo responsabilidad, ajustándose a las normas sobre barreras arquitectónicas y de accesibilidad (13). Asimismo, aprobar el plan de acondicionamiento territorial que identifique las áreas urbanas y de expansión urbana. Ejecutar directamente o proveer la ejecución de las obras de infraestructura urbana $\circ$ rural que sean indispensable para el desenvolvimiento de la vida del vecindario. La tabla 3 muestra las variables seleccionadas como inputs $u$ outputs, y que son consideradas pertinentes para la estimación de los niveles de eficiencia.

\section{Estimación del modelo}

Con las técnicas del DEA e índice de Malmquits se han realizado estimaciones para los 26 gobiernos locales de la muestra, procedimiento a partir del cual se obtiene la solución mostrada en la tabla 4. 
Tabla $N^{\circ}$ 5: Niveles de eficiencia de los programas sociales y seguridad ciudadana.

\begin{tabular}{ll}
\hline \multicolumn{1}{c}{ Outputs } & \multicolumn{1}{c}{ Inputs } \\
\hline Output 1: Casos en DEMUNA & Inputs 1: Vehículos (número de unidades \\
(número de casos atendidos). & destinadas a servicios sociales). \\
Output 2: Beneficiarios de & Inputs 2: Equipos de oficina (número de \\
programas sociales (número de & unidades). \\
beneficiarios: vaso de leche, club & Inputs 3: Serenazgo (número de efectivos). \\
de madres, comedores populares, & Inputs 4: Personal (número de profesionales, \\
wawa wasi, adulto mayor, & técnicos, auxiliares). \\
organizaciones juveniles). & Inputs 5: Medios de comunicación (teléfonos, \\
& celulares, etc.).
\end{tabular}

Tabla N 6: Índice de Malmquits - programas sociales y seguridad ciudadana (2009 - 2014).

\begin{tabular}{|c|c|c|c|c|c|}
\hline Malmquist & $2009=>2010$ & $2010=>2011$ & $2011=>2012$ & $2012=>2013$ & $2013=>2014$ \\
\hline El Tambo & 0,58078889 & 0,29476132 & 1,21538106 & 0,61616474 & 0,72440374 \\
\hline Huancayo & 0,41918915 & 1,04545523 & 1,28781802 & 0,61665869 & 0,86066497 \\
\hline Chilca & 0,29840759 & 1,67298888 & 0,62907796 & 0,81411337 & 2,02420858 \\
\hline Tarma & 1,63532823 & 1,15302482 & 0,92015394 & 0,58640682 & 0,92876293 \\
\hline Perené & 0,77634225 & 0,65219789 & 0,89392268 & 0,58842571 & 0,62320893 \\
\hline Pichanaqui & 0,48928703 & 0,42303043 & 8,20792985 & 0,56139931 & 0,69305005 \\
\hline Pangoa & 0,78571123 & 0,32315417 & 1,09400002 & 1,62192978 & 0,37591179 \\
\hline Satipo & 1,05842870 & 0,79577196 & 3,14926298 & 0,24691482 & 0,73335493 \\
\hline Chanchamayo & 0,37706567 & 1,13848703 & 0,28858157 & 0,60434760 & 1,80651977 \\
\hline San Ramón & 0,72159033 & 0,92659344 & 1,27253872 & 0,70171112 & 0,75074712 \\
\hline Chupaca & 1,29705294 & 0,45778322 & 1,19296526 & 0,53429528 & 0,51489457 \\
\hline La Oroya & 2,06428744 & 0,41728891 & 0,63675230 & 0,96207804 & 0,51243061 \\
\hline Jauja & 1,22518349 & 1,80703761 & 0,70100881 & 0,78351303 & 0,36978777 \\
\hline Río Tambo & 1,32723780 & 0,43479211 & 0,69344453 & 0,11915660 & 0,66717700 \\
\hline Mazamari & 0,36967085 & 0,39377277 & 0,85033885 & 0,36751791 & 1,71048717 \\
\hline Río Negro & 0,57009326 & 1,82554995 & 2,42111623 & 0,01000000 & 100 \\
\hline Concepción & 0,94431000 & 0,67444283 & 1,00092032 & 1,19157501 & 0,69312511 \\
\hline Junín & 1,09564012 & 0,91204208 & 1,58941188 & 0,28764670 & 1,09911298 \\
\hline Sapallanga & 0,19749587 & 100 & 0,26596078 & 0,19645891 & 97,05882130 \\
\hline Pilcomayo & 0,84748133 & 0,1575591 & 0,96346185 & 0,81829007 & 1,00474210 \\
\hline Huancán & 1,34458924 & 1,16362172 & 1,33787334 & 0,30555538 & 0,81149023 \\
\hline San Agustín & 0,41204832 & 1,80232443 & 0,40747420 & 0,61005560 & 0,48092567 \\
\hline Yauli & 0,20509882 & 2,52518401 & 1,70437358 & 0,52089125 & 0,58005702 \\
\hline Morococha & 1,16904313 & 0,62098757 & 1,15012203 & 0,63181318 & 1,04501220 \\
\hline Huay-Huay & 0,75780981 & 0,41066514 & 1,87635646 & 0,37467481 & 19,69976300 \\
\hline Santa Rosa de Sacco & 0,70184954 & 0,46257938 & 0,60933585 & 0,49455619 & 0,50537027 \\
\hline
\end{tabular}

A partir de la tabla 4 se puede identificar los siguientes comportamientos:

- La evolución del índice de Malmquist presenta una alta heterogeneidad, no se cuenta con un comportamiento eficiente de casi todos los gobiernos locales durante el período en la gestión de licencias de construcción y construcción de pistas y veredas, reafirmando la no existencia de una adecuada planificación de mediano y largo plazo.

- El gobierno local de Huancayo, en lo referido a licencias de construcción y construcción de pistas y veredas, es el único que durante el período muestra una mejora permanente y con una tendencia de crecimiento, en el 2009 con un índice de 0,78, para llegar al 2014 con un índice de 1,03.

- Los gobiernos locales de El Tambo, Satipo, Chupaca, pese a tener continuidad en la presencia de las mismas autoridades, no evidencian el fortalecimiento de sus procesos de gestión de manera eficiente.

Para el período analizado la gestión de licencias de construcción y construcción de pistas y veredas mediante 
el índice de Malmquist, se puede observar que:

- Los gobiernos locales de Pangoa, Satipo, Chanchamayo, Río Negro, Sapallanga, Pilcomayo, Santa Rosa de Sacco, aparecen en cuatro de un total de cinco oportunidades con niveles de eficiencia.

- Los gobiernos locales de Huancayo, Tarma, Pichanaqui, San Ramón, Jauja, Huancán, San Agustín, Morococha, aparecen en tres de un total de cinco oportunidades con niveles de eficiencia.

- Los gobiernos locales de El Tambo, Chilca, Chupaca, Río Tambo, Mazamari, Concepción, Junín, Huay-Huay, aparecen en dos de un total de cinco oportunidades con niveles de eficiencia.

- Los gobiernos locales de Perené, La Oroya, Yauli, aparecen en uno de un total de cinco oportunidades con niveles de eficiencia.

\section{Función de producción de los servicios sociales locales}

En esta competencia deben cumplir con regular acciones de las Defensorías Municipales de los Niños y Adolescentes (DEMUNA); ejecutar el Programa de Vaso de Leche (15); establecer canales de comunicación y cooperación entre los vecinos y los programas y organizaciones sociales; establecer un sistema de seguridad ciudadana, con participación de la sociedad civil y de la policía nacional. La tabla 5 muestra las variables seleccionadas como inputs $u$ outputs, y que son consideradas pertinentes para la estimación de los niveles de eficiencia.

\section{Estimación del modelo}

Con las técnicas del DEA e índice de Malmquits se han realizado estimaciones para los 26 gobiernos locales de la muestra, procedimiento a partir del cual se obtiene la solución mostrada en la tabla 6.

A partir de la tabla 6 se puede identificar los siguientes comportamientos:

- La evolución del índice de Malmquist vuelve a presentarnos un comportamiento heterogéneo, no se cuenta con un proceso eficiente en casi todos los gobiernos locales durante el período en la gestión de las DEMUNAS y los programas sociales, evidenciando una vez más la no existencia de una planificación de mediano y largo plazo.

- Es de destacar que los gobiernos locales de Chilca, Perené, Pichanaqui, Pangoa, Satipo, Chanchamayo, San Ramón, Chupaca, Río Tambo, Mazamari, Concepción, Junín, que según el INEI registran altos niveles de pobreza y pobreza extrema, ninguno de ellos presenta índices de Malmquist superiores a la unidad (indicador de eficiencia), entre ellos registran un promedio de 0,79 en el 2009 y 0,97 en el 2014, mostrando una tendencia creciente para el periodo.

- Los gobiernos locales de El Tambo, Satipo, Chupaca, pese a tener continuidad en la presencia de las mismas autoridades, no evidencian una política de gestión eficiente en materia de programas sociales.

- Los gobiernos locales capitales de las provincias, no marcan la diferencia pese a manejan mayor nivel de presupuesto, mayor personal y mayor infraestructura.

Para el periodo analizado la gestión de atenciones de casos en DEMUNA y beneficiarios de programas sociales mediante el índice de Malmquist, se puede observar que:

- Los gobiernos locales de Río Negro, Junín, Huancán, Morococha, aparecen en tres de un total de cinco oportunidades con niveles de eficiencia.

- Los gobiernos locales de Huancayo, Tarma, Pangoa, Satipo, Chanchamayo, Chupaca, Jauja, Concepción, Sapallanga, Yauli, Huay-Huay, aparecen en dos de un total de cinco oportunidades con niveles de eficiencia.

- Los gobiernos locales de Chilca, Pichanaqui, San Ramón, La Oroya, Río Tambo, Mazamari, Pilcomayo, San Agustín, aparecen en una de un total de cinco oportunidades con niveles de eficiencia.

- Los gobiernos locales de El Tambo, Perené, Santa Rosa de Sacco, no aparecen en ninguna de las cinco oportunidades con niveles de eficiencia.

\section{DISCUSIÓN}

La Ley Orgánica de Municipalidades (5) define a los gobiernos locales como "entidades, básicas de la organización territorial del Estado" que tienen la finalidad "representar al vecindario, promover la adecuada prestación de los servicios públicos locales y el desarrollo integral, sostenible y armónico de su circunscripción".

1. El crecimiento de la economía peruana se ve reflejado en presupuestos de los gobiernos locales de Junín. Estos gobiernos en el 2007 disponían de un presupuesto de 357 millones de nuevos soles, mientras que en el 2013 ascendió a 1098 millones mostrando un crecimiento del 207,5 \% (4) de manera que de carencia de recursos no 
padecieron.

2. En el caso del presupuesto para inversiones, en el año 2007 disponían en conjunto de 202 millones de nuevos soles y en el 2013 tenían 668 millones, presentando un crecimiento del $230,7 \%$, por lo que recursos para inversiones había. Los niveles de ejecución presupuestaria, en cambio, pasaron de $43,3 \%$ en el 2007 a $60,5 \%$ en el 2013.

3. La evaluación de los niveles de eficiencia aplicada a la competencia: servicios públicos locales (recolección y disposición final de residuos sólidos - tablas 1 y 2) se explica a partir de las variables: número vehículos destinados a la recolección de residuos sólidos; costos que genera la recolección de residuos sólidos; número de trabajadores destinados a la recolección de residuos sólidos:

- De los 26 gobiernos locales, solo en el período 2010 - 2011 se presentó que el 61,5\% (16 gobiernos locales) alcanzaron niveles de eficiencia, en los períodos restantes fueron más los gobiernos locales que muestran evidencia de ineficiencias.

- Gobierno locales como Huancayo, El Tambo, Chilca, que son ciudades con gran población y altos niveles de presupuesto, no han desarrollado sus funciones de servicios públicos locales (residuos sólidos) de manera eficiente (no aparecen en el grupo de gobiernos locales eficientes durante el período).

- Gobiernos locales como El Tambo, Satipo, Chupaca, que desde el 2007 al 2014 han tenido la misma autoridad edil, tampoco desarrollan un proceso de adecuación al tratamiento de los residuos sólidos con eficiencia, lo cual es muestra de una falta de planificación de mediano y largo plazo.

4. La evaluación de los niveles de eficiencia aplicado a la competencia: organización del espacio físico y uso del suelo (pistas y veredas, licencias de construcción - tablas 3 y 4) se explica a partir de las variables: número de maquinaria pesada; número de equipos de oficina; número de profesionales, técnicos, auxiliares; gasto en infraestructura:

- De los 26 gobiernos locales, en los períodos 2009 - 2010 y 2011 - 2012 se presentaron que el $38,5 \%$ (10 gobiernos locales) y $42,3 \%$ (11 gobiernos locales) alcanzaron niveles de eficiencia, evidenciando cierta aproximación a niveles de eficiencia adecuados en los otros períodos o quizás la "vocación" por solo "sembrar cemento" se cumple con las competencias de los gobiernos locales.
- La evolución del índice de Malmquist presenta una alta heterogeneidad, no se cuenta con una cultura y formación de eficiencia en todos los gobiernos locales durante el período en la gestión de licencias de construcción y construcción de pistas y veredas, reafirmando la no existencia de una adecuada planificación de mediano y largo plazo.

5. La evaluación de los niveles de eficiencia aplicado a la competencia: servicios sociales locales (número de casos en DEMUNA, número de beneficiarios de programas sociales, tablas 5 y 6 ) se explica a partir de las variables: número de vehículos destinados a servicios sociales; número de equipos de oficina; número de efectivos de serenazgo; número de profesionales, técnicos, auxiliares; medios de comunicación, teléfonos, celulares, etc.

- De los 26 gobiernos locales, solo en el período 2011 - 2012 se presentó que el 53,8 \% (16 gobiernos locales) alcanzaron niveles de eficiencia, en los períodos restantes fueron más los gobiernos locales que muestran evidencia de ineficiencias, lo cual evidencia un total desinterés por la atención de los temas sociales que son parte de las funciones de un gobierno local.

- Gobiernos locales como El Tambo, Perené, Santa Rosa de Sacco, no registran indicadores de eficiencia durante el período de estudio, lo cual muestra una carencia total de planificación de mediano y largo plazo.

- La evolución del índice de Malmquist se muestra altamente heterogéneo, no se cuenta con una cultura y formación de eficiencia en todos los gobiernos locales durante el período en la gestión de los servicios sociales locales.

6. Si bien autores como Chediak (1), Schuschny (6), Gonzales (10) y Horst (12), Ilegan a establecer unidades de toma de decisiones que luego sirven como referentes para que las otras unidades alcancen la eficiencia; en el caso de Junín, dado la heterogeneidad del comportamiento del índice de Malmquist durante el período de estudio 2009 - 2014, para el caso particular de las tres competencias que corresponden a los gobiernos locales de Junín, no es posible identificar unidades de toma de decisiones (DMU) de eficiencia que sirvan como referencia a estos gobiernos locales, limitando el cumplimiento de la misión institucional y de las competencia de los gobiernos locales, por lo tanto, postergando el desarrollo integral de su jurisdicción. 


\section{REFERENCIAS BIBLIOGRÁFICAS}

1. Chediak PF. La técnica DEA para medir y analizar la eficiencia de municipios del departamento de Tolima, 2006 [Tesis de maestría]. Pereira: Universidad Tecnológica de Pereira, Facultad de Ingeniería Industrial; 2008.

2. Ministerio de la Mujer y Desarrollo Social. Análisis de eficiencia del Programa Nacional Wawa Wasi a nivel de sedes zonales, período 2007, 2008 y 2009. Lima: MIMDES / Dirección de Monitoreo y Evaluación de Impacto Social; 2011.

3. Ministerio de la Mujer y Desarrollo Social. Análisis de eficiencia de los equipos de trabajo zonales del PRONAA en la gestión del Programa Integral de Nutrición, Caso Componente Alimentario. Período 2007, 2008 y 2009. Lima: MIMDES / Dirección de Monitoreo y Evaluación de Impacto Social; 2011.

4. Banco Central de Reserva del Perú. Memorias anuales. Lima: BCRP; 2007 - 2014.

5. Ley Orgánica de Municipalidades. Ley № 27972. Diario Oficial El Peruano, N 8373, (27-05-2003).

6. Schuschny A. El método DEA y su aplicación al estudio del sector energético y las emisiones de CO2 en América Latina y el Caribe. Santiago de Chile: CEPAL; 2007.

7. Cooper WW, Seiford LM, Tone K. Introduction to Data Envelopment Analysis and Its Uses, With DEA - Solver Software and References. New York: Springer; 2006.

8. Benedetti BC. Análisis y evaluación de la gestión educacional municipal [Tesis de pregrado].
Santiago de Chile: Universidad de Chile, Facultad de Ciencia Físicas y Matemáticas; 2010.

9. Gimenez GV, Prior JD. Evaluación frontera de la eficiencia en costes: Una aplicación a los municipios de Cataluña. VII Encuentro de Economía Pública. España: Universitat Autónoma de Barcelona; 2000.

10. González PR. Utilización del análisis envolvente de datos (DEA) en el desarrollo de una metodología para el establecimiento de costos eficientes de remuneración en la administración, operación y mantenimiento de los sistemas de distribución eléctrica [Tesis de postgrado]. Bogotá: Universidad Nacional de Colombia, Facultad de Ingeniería; 2010.

11. Tam MM. Una aproximación a la eficiencia técnica del gasto público en educación en las regiones del Perú. Lima: CIES; 2008.

12. Horst B. ¿Son nuestros municipios eficientes? Una aplicación del modelo de análisis envolvente de datos. Libertad y Desarrollo. 2007; 175(32): 3-30

13. Herrera CP, Francke BP. Análisis de la eficiencia del gasto municipal y de sus determinantes. Lima: Pontificia Universidad Católica del Perú; 2007.

14. Ligarda J, Ñaccha M. La eficiencia de las organizaciones de salud a través del análisis envolvente de datos: Microrredes de la Dirección de Salud IV Lima Este 2003. An Fac Med. 2006: 67(2); 142-151.

15. Pérez RK. Utilización del análisis envolvente de datos en la seguridad ciudadana. EAP de investigación operativa [Tesis de pregrado]. Lima: Universidad Nacional Mayor de San Marcos, Facultad de Ciencias Matemáticas; 2009. 